Voix et Images

voixetimages

\title{
Deux journalistes exceptionnelles
}

\section{Lori Saint-Martin}

Volume 18, numéro 3 (54), printemps 1993

Littérature, folie, altérité

URI : https://id.erudit.org/iderudit/201055ar

DOI : https://doi.org/10.7202/201055ar

Aller au sommaire du numéro

Éditeur(s)

Université du Québec à Montréal

\section{ISSN}

0318-9201 (imprimé)

1705-933X (numérique)

Découvrir la revue

Citer cet article

Saint-Martin, L. (1993). Deux journalistes exceptionnelles. Voix et Images, 18(3), 606-610. https://doi.org/10.7202/201055ar d'utilisation que vous pouvez consulter en ligne.

https://apropos.erudit.org/fr/usagers/politique-dutilisation/ 


\section{Féminismes}

\section{Deux journalistes exceptionnelles}

\section{Lori Saint-Martin, Université du Québec à Montréal}

On ne dira jamais assez l'importance des modèles. Pour les hommes désireux de se lancer en politique, en art ou en littérature, les modèles sont légion; les pères, source d'inspiration, s'imposent même par une présence parfois trop écrasante. Alors que les femmes, jusqu'à tout récemment - et encore - - souffraient plutôt d'une carence de modèles. Comment dire l'angoisse de celle qui doit s'imposer l'épreuve d'être la première, la seule, dans son domaine? Quel courage lui faut-il pour s'imaginer capable, elle, une femme, de réalisations dont on lui a dit qu'elles appartenaient aux seuls hommes? Ainsi, dès que des écrivaines s'interrogent sur la place des femmes dans la création, elles se tournent vers les grandes figures féminines du passé. Qu'il s'agisse de Christine de Pizan, au xve siècle, de Virginia Woolf au début du $\mathrm{xx}^{\mathrm{e}}$, ou des féministes de nos jours, les "mères littéraires. constituent un détour inévitable. Pour la journaliste Colette Beauchamp, la mère littéraire, le modèle, le phare, a été Judith Jasmin. Elle signe, de la prestigieuse journaliste, une biographie engagée, vivante, exceptionnelle ${ }^{1}$, proche par son esprit de la "biographie féministe de Simone de Beauvoir que nous a donnée Deirdre Bair. Elle a du courage, cette journaliste qui ose critiquer le monde du journalisme: «La presse a le privilège de se prononcer sur tous les secteurs d'activités humaines mais elle accepte mal qu'on lui rende la pareille ${ }^{2}$.

Drôle d'histoire que celle de Judith Jasmin: elle arrive dans le journalisme presque par accident, après avoir joué dans des radioromans et des pièces de théâtre, et a le bonheur de vivre les débuts de la télévision, moment de foisonnement collectif sur fond de contestation sociale, politique, culturelle. Elle accumule les primeurs: seule femme à la section française du Service international de Radio-Canada, seule femme, souvent, à joindre les rangs des équipes de tournage dépêchées à l'extérieur, première femme correspondante à l'étranger. Avec René Lévesque, au magazine radiophonique Carrefour et puis à la télévision, elle discute du chômage, des bas salaires, de l'avortement: Radio-Canada est alors le lieu privilégié des nouveaux intellectuels laïcs, à la pensée de gauche. Elle réalise, ici et à l'étranger, de grands reportages qui l'ont rendue célèbre. 
Pourtant, même une fois permanente, elle a toutes les peines du monde à obtenir la moindre augmentation de salaire; les reportages qu'elle tourne à l'extérieur à titre de pigiste lui sont payés à des cachets bien inférieurs à ceux qu'on offre aux hommes. Jamais on ne lui a proposé de poste de direction, malgré ses compétences; jamais on ne lui a confié l'animation en direct d'une émission d'affaires publiques, peut-être parce qu'on craignait son franc-parler. En 1966, elle se trouve même sans contrat de travail et doit se relancer à la pige, à cinquante ans, comme correspondante aux Nations unies, malgré sa réputation de journaliste hors pair; à la fin de sa vie, de retour à Montréal, elle se verra réduite à couvrir des défilés de mode et des dîners-causeries. Tout cela, elle l'accepte sans discuter, trop peu sûre d'elle pour exiger mieux.

En amour, elle sera toujours celle qui aimera davantage, et pour la vie. Dans ses histoires intimes avec René Lévesque ou le diplomate haïtien Joseph Châtelain, la grande journaliste se transforme en "amoureuse servile", traquée par l'insécurité affective. Comme si, malgré toutes les réalisations personnelles, elle croyait encore devoir, pour plaire, s'oublier au profit des autres. Conditionnement féminin bien ancré...

Engagée mais ouverte, jamais dogmatique, la biographie de Colette Beauchamp touche par sa générosité. Beaucoup de biographes se croient obligés de tuer symboliquement leur sujet (trop admiré?) en en révélant, avec une complaisance malsaine, les faiblesses cachées; chez Colette Beauchamp, la biographie est plutôt affaire de complicité, de connivence, sans verser pour autant dans la complaisance. Sans le moindre didactisme, passe une leçon de vie illustrant les difficultés qu'éprouve une femme, même exceptionnelle, à se tailler une place dans ce qui était à l'époque - et reste encore en grande partie aujourd'hui - un monde d'hommes.

Le cas Jasmin donne à réfléchir, au moment où les médias n'en ont que pour Kim Campbell. On pourrait croire que tout est réglé, car les journalistes débattent de ses compétences sans évoquer son sexe. Notons tout de même que les femmes politiques qui provoquent un tel engouement (on pense bien entendu à Margaret Thatcher) sont généralement de droite; les femmes de gauche dérangent bien davantage. Encore aujourd'hui, Judith Jasmin serait plus inquiétante pour les pouvoirs en place que madame Campbell. 
Pour accompagner la biographie, Colette Beauchamp a eu l'excellente idée de réunir en volume des textes de conférences et de reportages de Judith Jasmin ${ }^{3}$. Dès les années 1960 , la journaliste est du côté de toutes les bonnes causes: laïcisation de l'enseignement, mouvement antiraciste, pacifisme, écologie. Elle revendique pour tous les êtres de la terre, qu'il s'agisse des paysans algériens ou des Indiens du Grand Nord québécois, un traitement humain. Nulle trace pourtant d'orthodoxie, de dogmatisme, mais d'un parti pris en faveur de tous les êtres opprimés, d'un refus du statu quo et des stériles jeux de pouvoir. Convaincue que chacun, chacune a son rôle à jouer, Judith Jasmin agit et veut faire agir, pousse les gens à réfléchir et à participer au changement. La journaliste ne se place pas au-dessus des:autres, mais à leurs côtés, en plein combat.

La voix de Judith Jasmin, telle que ces textes nous la donnent à entendre, sonne clair et juste. Voix à la fois éminemment rationnelle (qui explique à merveille, en deux pages, les méandres de l'endettement du Tiers Monde, par exemple) et ouverte à l'émotion. Ton mesuré mais passionné, qui rappelle ce que Nicole Brossard a appelé "l'émotion de la pensée . Elle, une femme, parle de politique internationale, d'économie ou de désarmement, le plus naturellement du monde, comme si des femmes l'avaient toujours fait (alors qu'on sait la pression qui s'exerce encore, pendant ces années-là, pour que les femmes restent plutôt dans le silence du foyer), sans s'excuser, sans fausse modestie, sans non plus chercher à nous en mettre plein la vue, elle qui a voyagé et côtoyé les grands de ce monde. Sans renier non plus la différence des sexes, sans renier l'émotion, l'intérêt pour le quotidien, pour les femmes et les enfants, pour les petites gens en général, et sans se laisser piéger par l'Objectivité érigée en valeur absolue:

Je vous défie de faire un reportage sur le chômage, la délinquance ou la question raciale aux États-Unis en restant froid, neutre et objectif. Ou bien vous n'avez rien compris. Car comprendre, c'est aussi aimer, $s^{\prime}$ indigner, prendre parti, s'engager (p. 27) 4 .

À propos de condition féminine, les remarques de Judith Jasmin touchent et en même temps étonnent par leur naïveté. En 1952, elle écrit : "Est-ce à dire que le cycle des revendications est achevé de par le monde? En gros, oui. Les femmes sont admises à peu près partout" (p. 45). Au moment où elle affirme ainsi que tout est gagné ou presque, les femmes ne peuvent, au Québec, comme elle le signale elle-même, ni signer un contrat de vente ou d'achat, ni lancer une entreprise, sans l'autorisation de leur mari; il faudra attendre encore bien des années pour voir une seule femme députée, juré, sénatrice... 
Quant aux injustices subies par la femme au travail, dont elle-même avait une connaissance de première main, pas un mot. Circulent encore dans son discours des lieux communs vieux comme le monde («l'homme qui est fait pour les idées générales s'adapte mal au particulier " [p. 49]), en même temps que s'esquisse une autre vision, proche de celle des féministes pacifistes de notre époque, selon laquelle la femme, grâce à ses liens concrets avec les enfants et la vie matérielle, tiendrait davantage à la paix, de sorte que son influence * s'exercerait à la façon d'un élément modérateur autour des grands tapis verts où l'on joue si facilement avec les bombes" (p. 48). Et elle conclut en évoquant la responsabilité et la mission des femmes: "C'est à la femme d'aujourd'hui, à qui l'on a tout accordé, de prouver qu'elle est digne de toutes les revendications conquises.... (p. 52, je souligne). Plusieurs de ces idées ont vieilli, mais demeure un avertissement très clair: nous avons à nous garder aujourd'hui du même triomphalisme. Le progrès n'est pas, malheureusement, irréversible.

\section{$*^{*} *$}

À relire Le Silence des médias, étude du monde du journalisme écrit et électronique que Colette Beauchamp a-signée voilà quelques années - et qui faisait déjà d'elle la digne continuatrice de Judith Jasmin -, on se dit que, depuis les années 1950, presque rien n'a bougé. Non seulement le journalisme est-il devenu un spectacle plutôt qu'un travail d'information, "fast-food indigeste", "paquet de feuilles volantes détachées, lancées dans toutes les directions" (p. 15), transformation contre laquelle Judith Jasmin mettait déjà en garde dans les années 1960, mais la place accordée aux femmes n'est-elle guère plus large qu'autrefois. Concentrées aux basses échelles (documentalistes, recherchistes), exclues ou presque des métiers techniques ou spécialisés comme des fonctions les plus valorisées (rédaction des journaux, émissions matinales de radio), les femmes se voient confier les sujets les moins cotés '(société, éducation), alors que les hommes se réservent l'information politique et économique comme les sports, les matières les plus prestigieuses. Masculin, le journalisme l'est aussi pour ce qui est des activités couvertes. Les regroupements féministes ont toutes les peines du monde à s'y faire entendre, alors que les porte-parole des banques, des partis politiques, du Conseil du patronat s'y taillent la part du lion. Bref, des gens ordinaires, de leurs intérêts et de leurs activités communautaires, sociales, politiques, il est très peu question. 
Comment corriger la situation? Pas par la censure - bien qu'on accuse souvent les féministes de vélléités en ce sens: "Ce que les femmes et les minorités exigent, ce n'est pas de restreindre la liberté d'expression - elle l'est déjã - mais bien de l'élargir aux groupes qui n'en jouissent pas. (p. 171). Seule une concertation des femmes du milieu aurait des chances de réussir, affirme Colette Beauchamp; ce que Judith Jasmin n'a pu faire seule, les femmes modernes devront l'oser. On mesure aujourd'hui la grande solitude de cette pionnière. Arrivée plus tard, elle aurait reçu l'appui d'autres femmes journalistes; elle aurait peutêtre trouvé des hommes plus à sa mesure. On ne peut s'empêcher de croire qu'elle est venue trop tôt pour son propre bonheur. Mais peut-être est-elle arrivée à point nommé pour nous, à qui elle peut servir, comme pour Colette Beauchamp, de mère littéraire, de modèle, de phare.

1. Colette Beauchamp, Judith Jasmin, de feu et de flamme, Montréal, Éditions du Boréal, 1992.

2. Id., Le Silence des médias, Montréal, Éditions du Remue-ménage, 1987, p. 11.

3. Judith Jasmin, Défense de la liberté, textes recueillis et présentés par Colette Beauchamp, Montréal, Éditions du Boréal, coll. . Papiers collés., 1992.

4. On croirait entendre Gabrielle Roy, grande amie d'ailleurs de Judith Jasmin. 